\title{
The amendatory method of three dimensional rotary mechanisms
}

\author{
Shi Yan, Liu Zongzheng, Liu Xinmin, Han Hongwei \\ China Aerodynamics Research and Development Center, Mianyang 621000, China \\ Email: chinasyde@foxmail.com
}

\begin{abstract}
Keywords: the Captive Trajectory Simulation, homogeneous transformation matrix, coordinative control.

Abstract. First, the author measures the pose of the aircraft model under the help of Laser Tracker System; then, the author creates the amendatory equation on the bases of measuring; At last, the compensation value is input into the control system, a enhanced result can be got. The paper affirms the Captive Trajectory System can reach a satisfactory result by coordinative control of two revolve axis without improve the machining accuracy and assembly accuracy.
\end{abstract}

\section{Introduction}

Captive Trajectory Simulation (CTS) is an advanced and difficult technique used in wind tunnel testing. This technique is mainly used to assess the safety character when an extra component (e.g. extra fuel, extra weapon, and so on) detaches from its mother-aircraft. CTS technique is necessary for the development and enhancement of modern aircraft and the extra weapon. CTS system can simulate all its six dimensional movements (lift, drag, side, pitch, yaws and roll) when an extra component launches or detaches from the mother-aircraft. The system is compounded by six dimensional mechanism and corresponding control and measure system. As showed in figure 1, the base structure can move in lift, drag, and side direction, while the upper structure can move in three rotation direction, pitch, yaw and roll respectively. The upper structure is named Three Dimensional Rotary Mechanisms (TDRM). There are two push rods installed the left side and bottom of the mechanism. The two push rods can drive the mechanism in pitch and yaw directions. There is a hollow motor installed on the head, which can drive the mechanism in roll direction. The three dimensional rotary mechanism is the key part of the CTS system, and the system's precision relies on the TDRM.

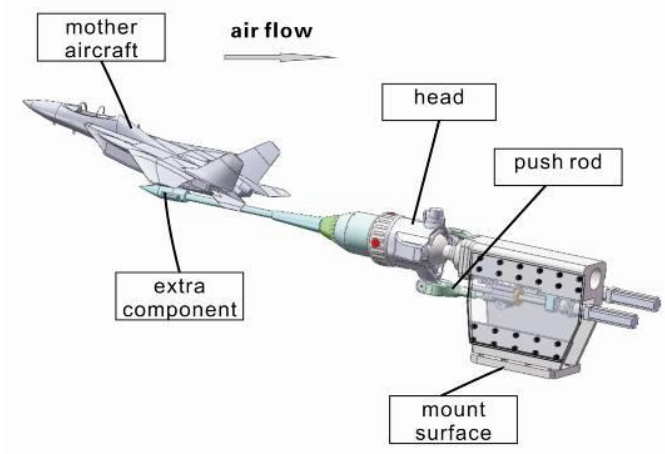

Figure 1 Three Dimensional Rotary Mechanisms

\section{The introduction and motion analysis of TDRM}

The TDRM is composed by spherical basement middle crust and outer crust, as showed in figure 2. The spherical basement is fixed on the rack, and can move synchronously with the rack in 
lift, drag and side directions. The middle crust is installed on the spherical basement, and can rotate along the vertical axis thus make a pitch movement. The outer crust is installed on the middle crust, and can rotate along the horizontal axis thus make a yaw movement. So, the TDRM can adjust the pitch and yaw angle of extra components. To record the angle, we install two encoders on the TDRM. One named $\alpha$ can record the relative angle between the rack and the middle crust, and the other named $\beta$ can record the relative angle between the middle crust and the outer crust. We define the pose of extra components as figure 3 with $\theta \sim$ pitch and $\Psi \sim$ yaw, so the angle pose $\mathrm{A}_{0}$ is $\left[\begin{array}{llll}\cos \theta_{0} \cos \psi_{0} & \sin \theta_{0} & \cos \theta_{0} \sin \psi_{0} & 0\end{array}\right]^{T}$

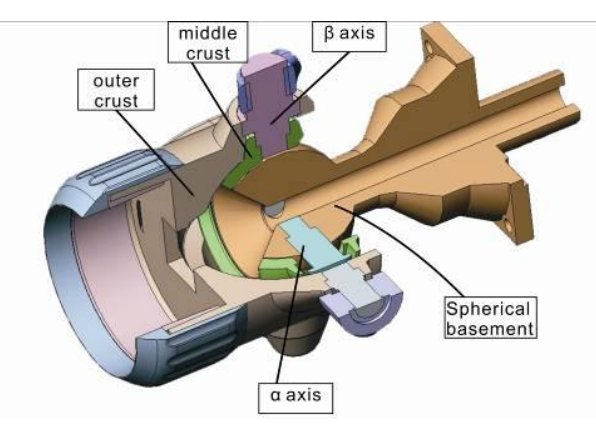

Figure 2 the head of TDRM

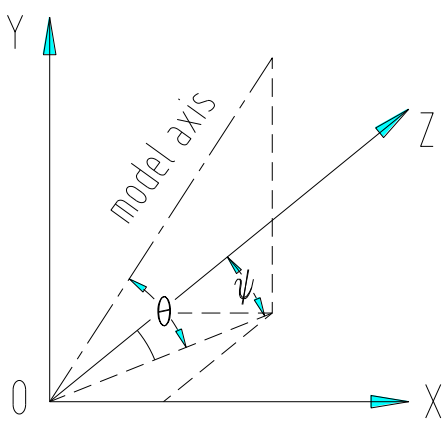

Figure 3 coordinate system

Assume that all the parts are manufactured and assembled in ideal condition and the extra components are installed precisely, the rotational operator matrices of the middle crust are:

$$
\operatorname{Rot}(y,-\beta)=\left[\begin{array}{cccc}
\cos (-\beta) & 0 & \sin (-\beta) & 0 \\
0 & 1 & 0 & 0 \\
-\sin (-\beta) & 0 & \cos (-\beta) & 0 \\
0 & 0 & 0 & 1
\end{array}\right]
$$

The rotational operator matrices of the outer crust is:

$$
\operatorname{Rot}(z, \alpha)=\left[\begin{array}{cccc}
\cos (\alpha) & -\sin \alpha & 0 & 0 \\
\sin \alpha & \cos \alpha & 0 & 0 \\
0 & 0 & 1 & 0 \\
0 & 0 & 0 & 1
\end{array}\right]
$$

$\alpha$ and $\beta$ can be read from the encoders. So the pose A of the extra component is

$$
\bar{A}=\operatorname{Rot}(y,-\beta) \operatorname{Rot}(z, \alpha) A_{0}
$$

Given we know the $\alpha$ and $\beta$, we can deduce the pose of the extra component. While it is inevitable to produce the volumetric errors in the process of manufacture and assembling. To simplify the matter, we only consider that all the rotating axises pass through the original point $O$ of reference coordinate system, and are not parallel with reference axises. The reference coordinate system is fixed to the spherical basement, and the original point $O$ is the centre of the sphere.

When the middle crust rotate along the spherical basement, we assume the vector of the rotating axis is $k \sim\left[\begin{array}{llll}k_{x} & k_{y} & k_{z} & 0\end{array}\right]^{T}$. So we can get the homogeneous transformation matrix.

$$
\operatorname{Rot}^{\prime}(k, \beta)=\left[\begin{array}{cccc}
k_{x} k_{x} \text { vers } \beta+c \beta & k_{y} k_{x} \text { vers } \beta-k_{z} s \beta & k_{z} k_{x} \text { vers } \beta+k_{y} s \beta & 0 \\
k_{x} k_{y} \text { vers } \beta+k_{z} s \beta & k_{y} k_{y} \text { vers } \beta+c \beta & k_{z} k_{y} \text { vers } \beta-k_{x} s \beta & 0 \\
k_{x} k_{z} \text { vers } \beta-k_{y} s \beta & k_{y} k_{z} \text { vers } \beta+k_{x} s \beta & k_{z} k_{z} \text { vers } \beta+k_{z} c \beta & 0 \\
0 & 0 & 0 & 1
\end{array}\right]
$$


In the formula: $c \beta=\cos \beta ; s \beta=\sin \beta$; vers $\beta=1-c \beta$.

When the outer rotate along the middle crust, we assume the vector of the rotating axis is $n \sim$ $\left[\begin{array}{llll}n_{x} & n_{y} & n_{z} & 0\end{array}\right]^{T}$; so we can get the homogeneous transformation matrix.

$$
\operatorname{Rot}^{\prime}(n, \alpha)=\left[\begin{array}{cccc}
n_{x} n_{x} \text { vers } \alpha+c \alpha & n_{y} k_{x} \text { vers } \alpha-n_{z} s \alpha & n_{z} n_{x} \text { vers } \alpha+n_{y} s \alpha & 0 \\
n_{x} n_{y} \text { vers } \alpha+n_{z} s \alpha & n_{y} n_{y} \text { vers } \alpha+c \alpha & n_{z} n_{y} \text { vers } \alpha-n_{x} s \alpha & 0 \\
n_{x} n_{z} \text { vers } \alpha-n_{y} s \alpha & n_{y} n_{z} \text { vers } \alpha+n_{x} s \alpha & n_{z} n_{z} \text { vers } \alpha+n_{z} c \alpha & 0 \\
0 & 0 & 0 & 1
\end{array}\right]
$$

In the formula: $c \alpha=\cos \alpha ; s \alpha=\sin \alpha ; \operatorname{ver} s \alpha=1-c \alpha$.

So we can deduce that the pose $\bar{A}^{\prime}$ of the extra component, when error occur.

$$
\bar{A}^{\prime}=E_{1}^{\prime} \operatorname{Rot}^{\prime}(k,-\beta) E_{2}^{\prime} \operatorname{Rot}^{\prime}(n, \alpha) A_{0}
$$

In the formula: $E^{\prime}{ }_{1}$ is the original pose of the middle crust and $E^{\prime}{ }_{2}$ is the original pose of the outer crust.

\section{The test for mechanical precision}

The encoders installed on the horizontal axis and the vertical axis can recode the relative angles, and send the vale to the control system. So it is possible to precisely control the mechanism.During the test, an laser tracker system was used to measure the pose of the extra component, including pitching angle $\theta$ and yawing angle $\Psi$.

In ideal condition, that is to say, the horizontal axis and the vertical axis are mounted with no bias during the manufacture and assembling. When initial pitching angle $\theta$ and yawing angle $\Psi$ are set to $O$ respectively. When the horizontal axis is fixed during the test, the centre crust do not revolve along the spherical basement, outer crust revolves along the centre crust. that will cause change in pitching angle $\theta$ only. While the result is like figure 4 and figure 5 . The transverse axis represent the angle that the outer crust revolves along the middle crust. That angle is equal to $\beta$. The longitudinal axis in figure 4 represents the angle difference between $\beta$ and $\Psi$. The longitudinal axis in figure 5 represents the angle $\theta . \theta$ and $\Psi$ can be read from laser tracker system.

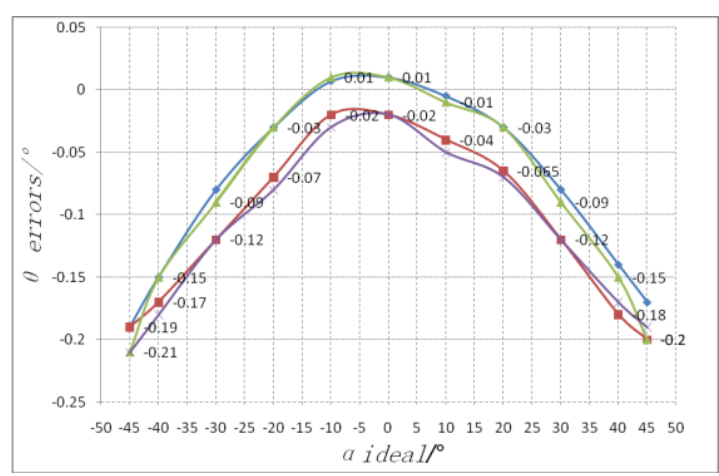

Figure 4

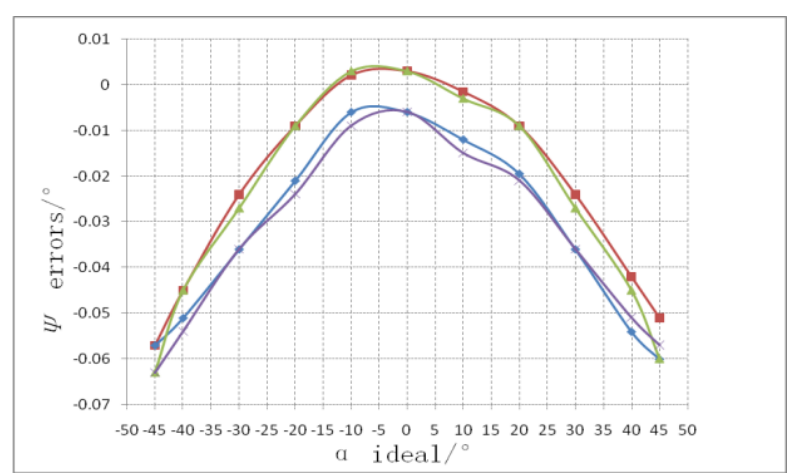

Figure 5

When the vertical axis is fixed during the test, the outer crust do not revolve along the outer crust, the two revolves along the spherical basement together. That will cause change in yawing angle $\Psi$ only. While the result is like figure 6 and figure 7. The transverse axis represents the angle that the two revolve along the spherical basement. The angle is equal to $\alpha$. The longitudinal axis in figure 6 represents the angle difference between $\alpha$ and $\theta$. The longitudinal axis in figure 7 represents the angle $\theta . \theta$ and $\Psi$ can be read from laser tracker system. 


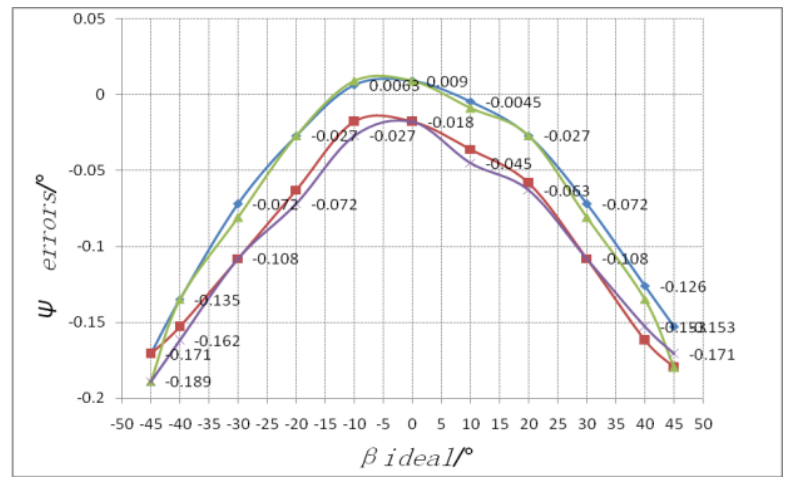

Figure 6

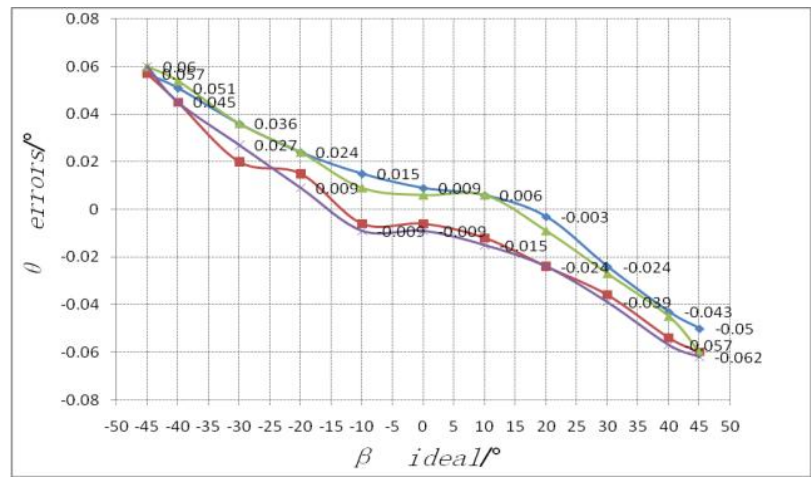

Figure 7

From the figure 4 7 and equation (6), the vector of the rotating axis $k$ and $n$ can be deduced. Since $k$ and $n$ is known, it is easy to get extra component's pose that is wanted by inverse kinematics method. By that mean, the input $\alpha$ and $\beta$ can be attained. The figure 8 and 9 show the case that the errors decreases to one third of the original case compare to figure 4 and 5.

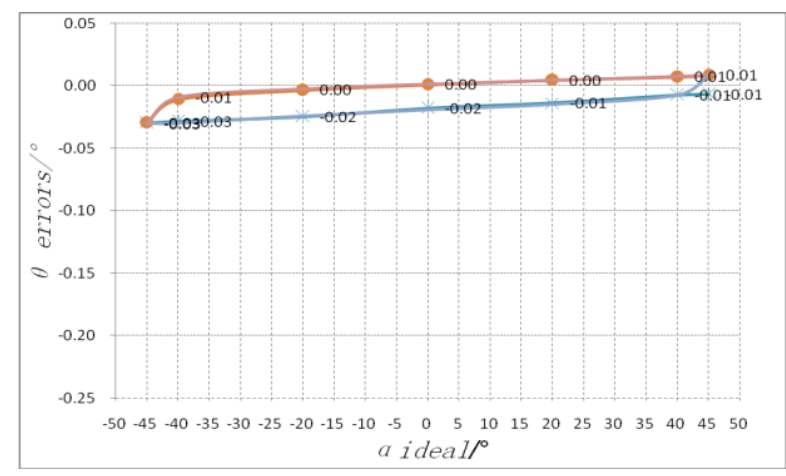

Figure 8

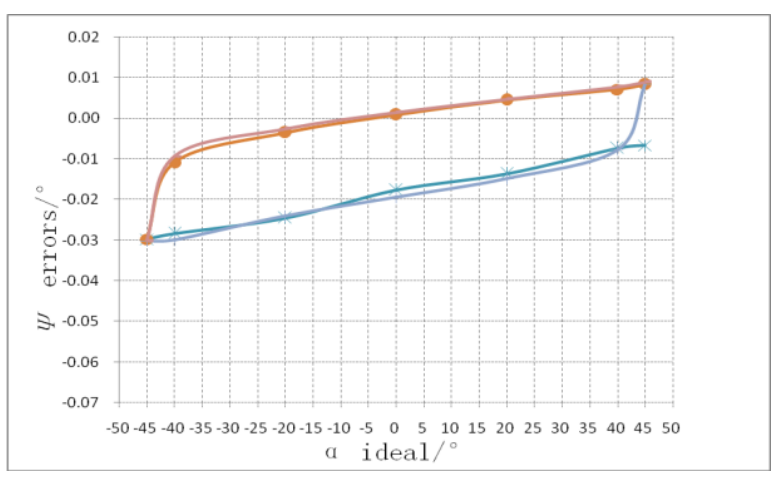

Figure 9

\section{Summary}

One can get the input of the joints through inverse kinematics method. The example listed in the paper is different to inverse kinematics. In the example, one knows not only the final position and pose but the input of the joints (the value can be read by encoder means). so the spin vectors $k$ and $n$ can be deduced. Then, one can gain revised input value by inverse kinematics method to get desiring pose.

Furthermore, in CTS testing, one can build motion transformation matrix equation and use Laser Tracker system to measure the mounted errors of the two axises. Then one get motion compensation. The test shows the model mounted on the top of the mechanisms can reach ideal position and pose by coordinate the motion of the two axises without enhance machining and mounting accuracy.

\section{References}

[1] HUANG Xu-hui, PANG Xu-dong. Development of a new captive trajectory simulation system in the $1.2 \mathrm{~m}$ transonic wind tunnel. Journal of Experiments in Fluid Mechanics. Jun. 2008:Vol.22,No.2.

[2] Xiong You-lun, WANG Zheng-jia: Industria Robot (version 2). [M].Wuhan: Huazhong science and technology university press, 2006 p26-32. 